\title{
Socially learned habituation to human observers in wild chimpanzees
}

Liran Samuni 1,2,5

Roger Mundry 1

Joseph Terkel 2

Klaus Zuberbühler 3,4,5

Catherine Hobaiter 3,5,*

Email clh42@st-andrews.ac.uk

1 Max Planck Institute for Evolutionary Anthropology, Leipzig, Germany

2 Department of Zoology, University of Tel Aviv, Tel Aviv, Israel

3 Centre for Social Learning and Cognitive Evolution and Scottish Primate Research Group, School of Psychology and Neuroscience, University of St Andrews, St Andrews, KY16 9JP Scotland, UK

4 Department of Comparative Cognition, Institute of Biology, University of Neuchatel, 2000 Neuchâtel, Switzerland

5 Budongo Conservation Field Station, P.O. Box 362, Masindi, Uganda

\section{Abstract}

Habituation to human observers is an essential tool in animal behaviour research. Habituation occurs when repeated and inconsequential exposure to a human observer gradually reduces an animal's natural aversive response. Despite the importance of habituation, little is known about the psychological mechanisms facilitating it in wild animals. Although animal learning theory offers some account, the patterns are more complex in natural than in laboratory settings, especially in large social groups in which individual experiences vary and individuals influence each other. Here, we investigate the role of social learning during the 
habituation process of a wild chimpanzee group, the Waibira community of Budongo Forest, Uganda. Through post hoc hypothesis testing, we found that the immigration of two well-habituated, young females from the neighbouring Sonso community had a significant effect on the behaviour of non-habituated Waibira individuals towards human observers, suggesting that habituation is partially acquired via social learning.

$\mathrm{AQ1}$

$\mathrm{AQ} 2$

\section{Keywords}

Female transfer

Human observation

Pan trogtodytes schweinfurthit

Social referencing

Social learning

Culture

mimitation

Dispersal

Observational conditioning

Electronic supplementary material

The online version of this article (doi:10.1007/s10071-014-0731-6) contains supplementary material, which is available to authorized users.

\section{Introduction}

Studying the behaviour of wild animals in their natural environment requires procedures to minimize the effect of the observer's presence. One such procedure is habituation (Williamson and Feistner 2011), a process by which repeated exposure to one or more human observers results in a continuous reduction in an animal's normally fearful responses to their presence. Full habituation is achieved when the study animals treat the observers as neutral elements in their environment (Schaller 1963; Magurran and Girling 1986; Tutin and Fernandez 1991). Despite its importance in animal behaviour research, little is known about the mechanisms underlying habituation in wild animals (Jack et al. 2008).

The term habituation is well established in animal learning theory, 
commonly defined as a simple non-associative learning process that is contrary to sensitization (Thorpe 1963; Stein 1966; Petrinovich and Patterson 1981; Mackintosh 1987). The main body of research on habituation consists of controlled laboratory manipulations that have measured responses to novel and familiar stimuli, typically in rodents and other small mammals (Mackintosh 1987). In invertebrates, the neurophysiological processes that underlie habituation have also been described (Burrell and Sahley 2001). Nonetheless, few studies have focused on habituation conducted in the animal's natural environment, despite the evolutionary relevance of the process (Raderschall et al. 2011). For wild animals, the tacit assumption has always been that habituation to human observers is, at least partly, caused by the same non-associative learning mechanism as described for laboratory animals. However, the social environment of wild animals is very different from the conditions under which habituation is typically studied in psychological laboratories (Raderschall et al. 2011). Importantly, wild animals have active control over their environments and can flexibly influence their exposure to external events, such as the presence of humans. Equally important, animals are able to learn socially from each other, suggesting that habituation in social groups may also be driven by processes of social learning.

There is considerable debate on the existence and nature of social learning in non-human animals (Whiten and Ham 1992). Various mechanisms have been discerned within social learning, including imitation, stimulus enhancement, observational conditioning, and emulation (Sherry and Galef 1984; Mineka et al. 1984; Tomasello 1990; reviewed by Whiten and Ham 1992 ). Research on social learning has a distinguished history, starting with early reports based on anecdotal observations of natural behaviour (e.g. Romanes 1882). The complex nature and diversity of socially acquired behaviours then shifted the field towards experimental work, usually by employing a variation of Thorndike's classic 'puzzle box' task (1898). In this task, a subject is allowed to observe a demonstrator, who has been previously trained to solve a complex task in order to receive a reward. The observer is then tested for success, speed, and similarity of its behaviour relative to a control animal that has had no opportunity for social learning. In more recent versions of this task, the subject observes either (a) an alternative way to solve the same task, or (b) a 'ghost control' in which the task is solved automatically without a demonstrator (e.g. Tomasello et al. 1987; Heyes and Dawson 1990; Akins and Zentall 1996; Custance et al. 
1999; Tennie et al. 2006).

Over the years, a substantial body of work on social learning among a wide range of species has accumulated, and it is therefore rather surprising that the precise nature of social learning abilities is still hotly debated (Whiten and Ham 1992; Caldwell and Whiten 2002). More critically, Thorndike's classic 'puzzle box' task and all its variations bear little resemblance to the conditions under which social learning takes place in the real world, in which inexperienced individuals learn the important features of their environment, such as how to find food and avoid predators, from observing others. Although socially acquired behaviour has been studied in wild populations, the priority has been to demonstrate cultural variations (Kawai 1965; McGrew and Tutin 1978; Nishida 1987), without considering the possible mechanisms by which these variations might be transmitted (cf: Byrne and Byrne 1993; Hobaiter and Byrne 2010 ).

We addressed this problem through a field study on habituation in wild chimpanzees. Apart from the theoretical issues outlined above, understanding the mechanisms underlying habituation is also important both for methodological reasons and for various applied issues in conservation biology. For example, it is often important to make accurate predictions about when a study group will be suitably habituated for behavioural research. Species, social organization, habitat structure, population density, and previous experience with humans are likely to play a role in the rate of progress (Ghiglieri 1984; Tutin and Fernandez 1991; Johns 1996). In nonhuman primates, habituation periods range from hours (prosimians), to months (forest monkeys), to years (great apes) (Williamson and Feistner 2011). In chimpanzees (Pan troglodytes), habituation is particularly timeconsuming. Large and usually densely forested home ranges, complex fission-fusion social structure, and a long history of conflict with humans, make this species relatively difficult to habituate in comparison with other primates (Morgan and Sanz 2003; Bertolani and Boesch 2008). In one well-documented case, the North community of Taï Forest, Côte d'Ivoire, chimpanzees were only sighted for $1 \%$ of observation time during the first 2 years of habituation (Boesch and Boesch 1990). Similarly, at the Gashaka Gumti National Park, Nigeria, wild chimpanzees were only observed for approximately $1.5 \%$ of observation time during the first 2 years of habituation, although patrols were not conducted on a daily basis (Sommer et al. 2004 ). In Budongo Forest, Uganda, habituation of the Sonso 
community was also slow. Beginning in 1990, individuals were located by auditory cues and tracked on a daily basis, so that by late 1994, 46 individuals could be identified and some of them followed individually (Newton-Fisher 1999).

Habituation may also have effects on the wider population of a species (Goldsmith 2005). For example, it has been suggested that habituation can 'spread' beyond the target group due to migration (Goldsmith 2005). In chimpanzees, this could result from young females dispersing to neighbouring communities (Nishida and Kawanaka 1972; Pusey 1979; Williams 1999) and, in doing so, transmitting their altered behavioural responses to humans to their unhabituated conspecifics.

We addressed this hypothesis through a study on habituation in the Waibira chimpanzee community of Budongo Forest, Uganda, which are direct neighbours of the already habituated Sonso community (Reynolds 2005 ). We tested a number of possible factors that could potentially influence habituation, measured as proximity to and duration of stay with an observer. In particular, we documented the habituation effects due to two adult nulliparous females, who had grown up with human observers in the Sonso community and later immigrated to the Waibira study group prior to the latter's habituation.

\section{Materials and methods}

\section{Study site and data collection}

Field research was conducted at the Budongo Conservation Field Station $\left(1^{\circ} 37^{\prime}-2^{\circ} 03^{\prime} \mathrm{N}, 31^{\circ} 22^{\prime}-31^{\circ} 46^{\prime} \mathrm{E}\right)$ in the Budongo Forest Reserve, in western Uganda. The $793-\mathrm{km}^{2}$ reserve comprises $482 \mathrm{~km}^{2}$ of continuous, medium altitude, semi-deciduous forest cover (Eggling 1969) with an estimated population of around 600 chimpanzees (Plumptre et al. 2003).

Habituation of the Waibira community started in March 2011, following a survey of a wider area surrounding the Sonso community. The strategy was to locate community members by their long-distance signals, particularly pant-hooting and drumming, and by waiting at potential fruiting trees and nesting areas. During chimpanzee encounters, we used 5-min interval scan sampling (Altmann 1974) of all individuals within sight to determine (a) party size and composition (age class and sex), (b) behavioural activity, (c) 
food species and part, (d) horizontal and vertical distance to observers, (e) GPS location, and (f) vocalizations produced. Data were collected using a hand-held HP iPaq computer, a Garmin GPS Map 60CSx, and a Panasonic HDC-SD60 high-definition camcorder to facilitate individual identification and to document key behaviours and interactions.

\section{Presence of the habituated females}

During the first few weeks of habituation (March-April 2011), we sighted two habituated adult nulliparous females, Nora and Bahati, who had immigrated from the neighbouring Sonso community into the Waibira community. Nora was born in February 1996 to Nambi, the alpha female of the Sonso community. Bahati was born in December 1994 to another core female, Kalema. DNA testing confirmed both as paternal siblings, sired by the male Black (Reynolds 2005). Both mothers had been identified from the start of detailed data collection in Sonso (1990), and had since been seen on an almost daily basis. As a consequence, Nora and Bahati had been exposed to human presence since early infancy and were fully habituated at the time of their dispersal in 2010.

We collected data on observation duration (min) and horizontal distance (m) to observer(s) of non-habituated Waibira community members during all chimpanzee encounters, which allowed for accurate individual identification and data collection, while noting the presence or absence of the habituated females among the observed party. In every encounter, we tried to maintain the closest position possible for observation while causing minimal interference with the chimpanzees' natural behaviour. During this process, we recorded subjects' responses to our presence. If an individual persistently monitored the observer, gave alarm vocalizations, moved in agitated ways, or produced distinctive self-scratching, we increased the observation distance until these behaviours stopped. At times, it was even necessary to remove ourselves completely from visual contact or to leave the party for a eertain time.

Since observation distance was strongly affected by a chimpanzee's vertical position within the canopy and need to consume essential resources, such as water or attached food items, we decided to restrict our analyses to encounters in which individuals were on the ground and engaged in activities that did not prevent them from moving without loosing any resources. Observation distances of chimpanzees in trees are highly 
variable, while observation conditions are heavily contained by the structure of the vegetation. For example, it is rarely possible to observe an individual by standing directly under the tree. Instead, a suitable gap in the canopy must be found, which is largely determined by the surrounding trees in a dense semi-deciduous secondary forest. In contrast, observation distances on the ground are closely linked to the minimally tolerated distance by the subject. When following chimpanzees on the group, we never approach closer than $7 \mathrm{~m}$, for welfare and health reasons (www.budongo.org).

Although they are adept climbers, Budongo chimpanzees spend much of their time on the ground (females $27 \%$, males $42 \%$; Kosheleff and Anderson 2008), where observation conditions are best, suggesting that restricting data collection on the ground generates a sufficiently large sample. For these reasons, we consider that observation of individuals on the ground to be the 'gold-standard' measure of chimpanzee habituation for the purpose of scientific observation.

\section{Effects of habituated females on horizontal distance to observer}

To determine whether the presence of one or both of the two habituated females had an effect on the other individuals' preferred distance $(\mathrm{m})$ to a human observer, we ran a generalized linear mixed model (GLMM; Baayen 2008 ). Into the model, we included the presence of one or both of the habituated females (no/yes), date (number of days elapsed since the beginning of the study), age class (non-adult/adult), and sex of the observed individual as fixed effects. Party size was also included as a fixed effect, as large parties are likely to enhance perceived safety-in-number effects (Hamilton 1971), leading to greater levels of tolerance towards observers. We included the interaction between the presence of a habituated female and date into the model, to test whether habituated females had stronger effects on habituation at the beginning than at the end of the observation period. As random effects, we included the individual's identity, its activity, the date, and the party identity (identifying consecutive observations of the exact same party). Furthermore, in order to keep type I error rate at the nominal $5 \%$, we included random slopes for the presence of the habituated females, the date, and the interaction between these two variables within individual and within party identity into the model (Baar et al. 2013 ). We did not include the correlation between random slopes and random intercepts as otherwise the model did not converge. This was 
necessary, on the one hand, because non-convergence of a model can seriously affect its validity and possible, on the other hand, since Baar et al. (2013) showed that not including the correlation between random intercepts and random slopes does not seriously compromise type I error rate. Before running the model, we $z$-transformed the variable date and group size (to a mean of zero and a standard deviation of one) and centred (to a mean of zero) the dummy coded presence of the habituated females $(0,1)$ to enable interpretation of the main effects despite them being involved in the interaction (Schielzeth 2010).

As the response variable, we entered the observed individual's ground distance from the human observer $(\mathrm{m})$. Although continuous by nature, we recorded distance as integer estimates in whole metres. As a result, the final model was implemented with Poisson error distribution and log link function. When several subjects within the same party were observed at the same distance, we randomly selected a single individual as the focal animal to avoid pseudoreplication (Hurlbert 1984). We repeated this random selection 1,000 times and presented the average of the results.

The influence of the habituated females was represented by two effects; their presence and their presence in interaction with the date. We thus compared the full model (as described above) with a null model that did not comprise these two effect terms but was identical to the full model in all other terms, keeping the probability of false positives at a desired error level $(\alpha=0.05$; Forstmeier and Schielzeth 2011). This was done using a likelihood ratio test (Dobson 2002).

Neither colinearity (Quinn and Keough 2002; Field 2005), as indicated by the largest variance inflation factor $(\mathrm{VIF}=1.08)$, nor overdispersion (dispersion parameter $=0.54, \chi^{2}=303.16, d f=558, P=1$ ) was an issue.

The model was implemented in $\mathrm{R}$ (version 3.0.1; R Core Team 2013) using the function lmer of the $\mathrm{R}$ package lme4 (Bates et al. 2013). VIF values were derived using the function vif of the R package car (Fox and Weisberg 2011 ) applied to a standard linear model comprising neither the random effects nor the interaction. The sample size for this model was 565 observations (scan sample data points) from 25 individuals (of which, due to the random selection, not all were included in each model) in 80 parties from 53 days. 


\section{Effects of habituated females on observation duration}

To determine whether the presence of the habituated females had an effect on observation duration (min) of the different individuals on the ground, we ran a GLMM with a Gaussian error structure and an identity link function, using the same encounters as for the previous distance analysis. The resulting duration model was identical to the above described distance model, apart from exclusion of the fixed effects of age class and gender, as well as the random effects of activity, since these factors had shown no significant effects in the model for distance. In addition, party identity was not included as a random effect as we had only one observation duration data point per party composition. Again, we included random slopes for date, the presence of the habituated females and their interaction into the model and this time also the correlation between these and the respective random intercept (Baar et al. 2013).

The response variable, observation duration (min), was log transformed. Again, we tested the significance of the presence of the immigrant females on observation duration, by comparing the deviance of the full model with that of a corresponding null model (i.e. excluding the presence of the habituated females and the interaction), using a likelihood ratio test. As for the previous model, if party size (excluding the two habituated females) was greater than one, we randomly selected a single individual from the party, repeated the random selection 1,000 times, and presented the average results. To obtain reliable $P$ values for the individual effects, we used likelihood ratio tests (Baar et al. 2013). The sample size for this analysis was a total of 83 parties observed at 54 days and including 25 individuals (of which, due to the random selection, not all were included in each of the 1,000 random selections).

\section{Assessment of model stability}

We determined the overall model stability for the two models (observation duration and distance). The stability of the model for distance from observer was assessed by excluding subjects $(N=25$ subjects $)$ and parties $(N=80)$ one by one, while in the observation duration model, we excluded only subjects one by one. To avoid confounding of subject exclusion with the random selection of subjects from parties, we excluded each subject 10 times using different random selection of subjects from parties. With this, we repeated the analysis and compared the results (estimated coefficients 
and $P$ values) with those revealed for all data.

\section{Results}

\section{Habituation and sample size}

Throughout the full study period from March 2011 to August 2012, we observed chimpanzees approximately $33 \%$ of tracking time $(N=1,470$ observation hours). The daily success rate of finding at least one chimpanzee (not including the habituated females) was $87 \%$. However, during the first 6 months of habituation (March-August 2011), it was not yet possible to reliably identify a sufficient number of individuals. We therefore restricted data analysis to the subsequent 12-month period (September 2011-August 2012), based on $N=25$ identified independent individuals (males: $N=20$; females: $N=5$ ). During this period, we observed at least one individual $37 \%$ of the tracking time $(N=1,120$ observation hours), with an average daily $96 \%$ success rate of finding at least one chimpanzee. In total, we documented 83 encounters with nonhabituated individuals on the ground that met the criteria for analysis, yielding 565 scan samples (at 5 min intervals). One or both of the immigrant females were present in $32.5 \%$ of encounters.

\section{Distance of non-habituated individuals from observer}

To assess the effects of the presence of other individuals on a nonhabituated individual's response to a human observer, we examined two social effects: party size and the presence of the habituated individuals.

Party size had a positive effect on the observation distance of nonhabituated individuals: horizontal distance between non-habituated individuals and the observer increased with increasing group size (Table 1, largest $P$ value across 1,000 random selections out of the parties: 0.017$)$. In contrast, the presence of the habituated individuals had a negative effect on observation distance, with the distance between non-habituated individuals and the observer decreasing in the presence of the habituated females (full null model comparison using a likelihood ratio test, average of 1,000 random selections: $\chi^{2}=19.77, d f=2, P<0.001$; all $P$ values in the assessment of model stability $\leq 0.001)$.

Table 1 
Mean results (averaged across 1,000 random selections of individuals) of the GLMM with horizontal distance from the observer as the response

\begin{tabular}{|c|c|c|c|c|}
\hline Term & Estimate & $\mathbf{S E}$ & $z$ & $\boldsymbol{P}$ \\
\hline Intercept & 3.085 & 0.074 & a & a \\
\hline Party size & 0.056 & 0.020 & 2.856 & 0.005 \\
\hline Age $(0=$ adult, $1=$ non-adult $)$ & -0.029 & 0.033 & -0.884 & 0.415 \\
\hline $\operatorname{Sex}(0=$ female, $1=$ male $)$ & 0.006 & 0.068 & 0.078 & 0.671 \\
\hline Date $^{b}$ & -0.164 & 0.036 & -4.504 & $<0.001$ \\
\hline Presence $^{c}$ & -0.303 & 0.059 & -5.146 & $<0.001$ \\
\hline Presence $^{c} \times$ date $^{b}$ & 0.151 & 0.078 & 1.936 & 0.054 \\
\hline \multicolumn{5}{|c|}{ anot indicated because of having no meaningful interpretation } \\
\hline \multicolumn{5}{|c|}{$\mathrm{b}_{z}$-Transformed to a mean of 0 and a standard deviation of 1} \\
\hline
\end{tabular}

The average across all random selections of subjects out of parties $(1,000$ repeats) revealed a borderline interaction between the presence of the habituated females and date (average $P=0.054$; range of $P$ values 0.018 0.099), suggesting that the effect of the habituated females was stronger at the beginning of the habituation process (Fig. 1; Table 1). We found no obvious effect of neither age $(P=0.415)$, nor sex $(P=0.671$; Table 1$)$; however, these two latter parameters were associated with large uncertainty.

\section{Fig. 1}

Distance from observer ( $\mathrm{m}$ ) in the presence (top) and the absence (bottom) of the habituated females. The area of the circles reflects the number of data points at the same respective distance and date 


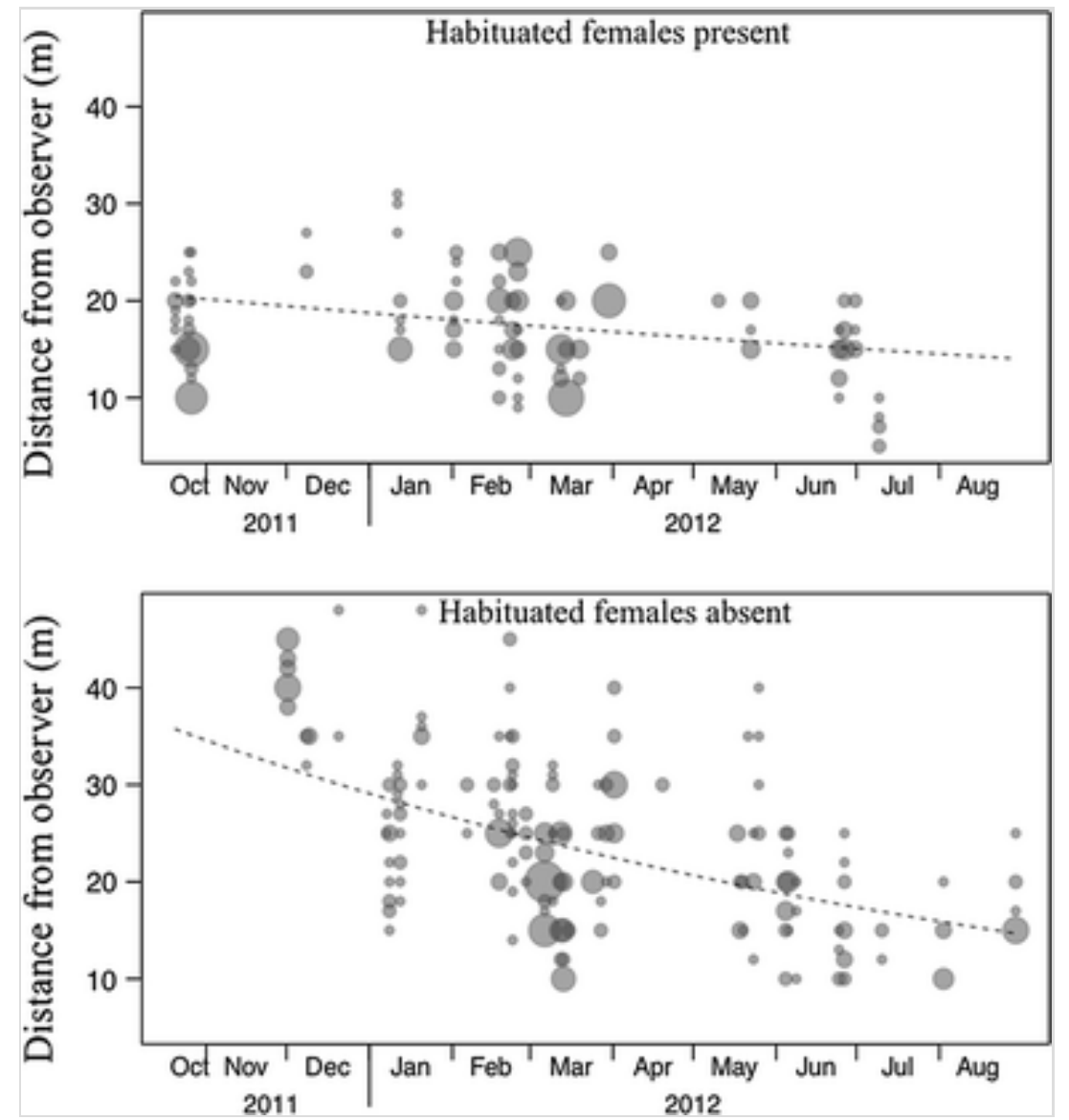

With regard to model stability, we found a robust impact of the presence of the two habituated females (difference between the largest and smallest $P$ values revealed for the full null model comparison $<0.0001$ ), without any obviously influential subjects or parties. We found the largest uncertainly for estimated coefficients that were not significant, but the interaction between date and the presence of the habituated females was also associated with some uncertainty (ESM Table S1).

\section{Observation duration of non-habituated individuals}

We found a clear effect of both party size and the presence of the habituated females (full null model comparison, average across the 1,000 random selections of subjects out of parties: $\chi^{2}=8.33, d f=2, P=0.021$; Fig. 2). After removal of the non-significant interaction $\left(\chi^{2}=2.26, d f=1\right.$, $P=0.152$ ), we found that observation duration of non-habituated individuals increased when the habituated females were present (average $P$ value across 1,000 random selections out of parties $=0.021)$ and with increasing party size $(P<0.001$; Table 2$)$. However, date did not have an effect on observation duration $(P=0.562)$.

Fig. 2 
Observation duration: shown are medians (dark horizontal lines), quartiles (boxes), percentiles (2.5 and $97.5 \%$; vertical lines), minimum and maximum (laying crosses), as well as the fitted model (grey lines)

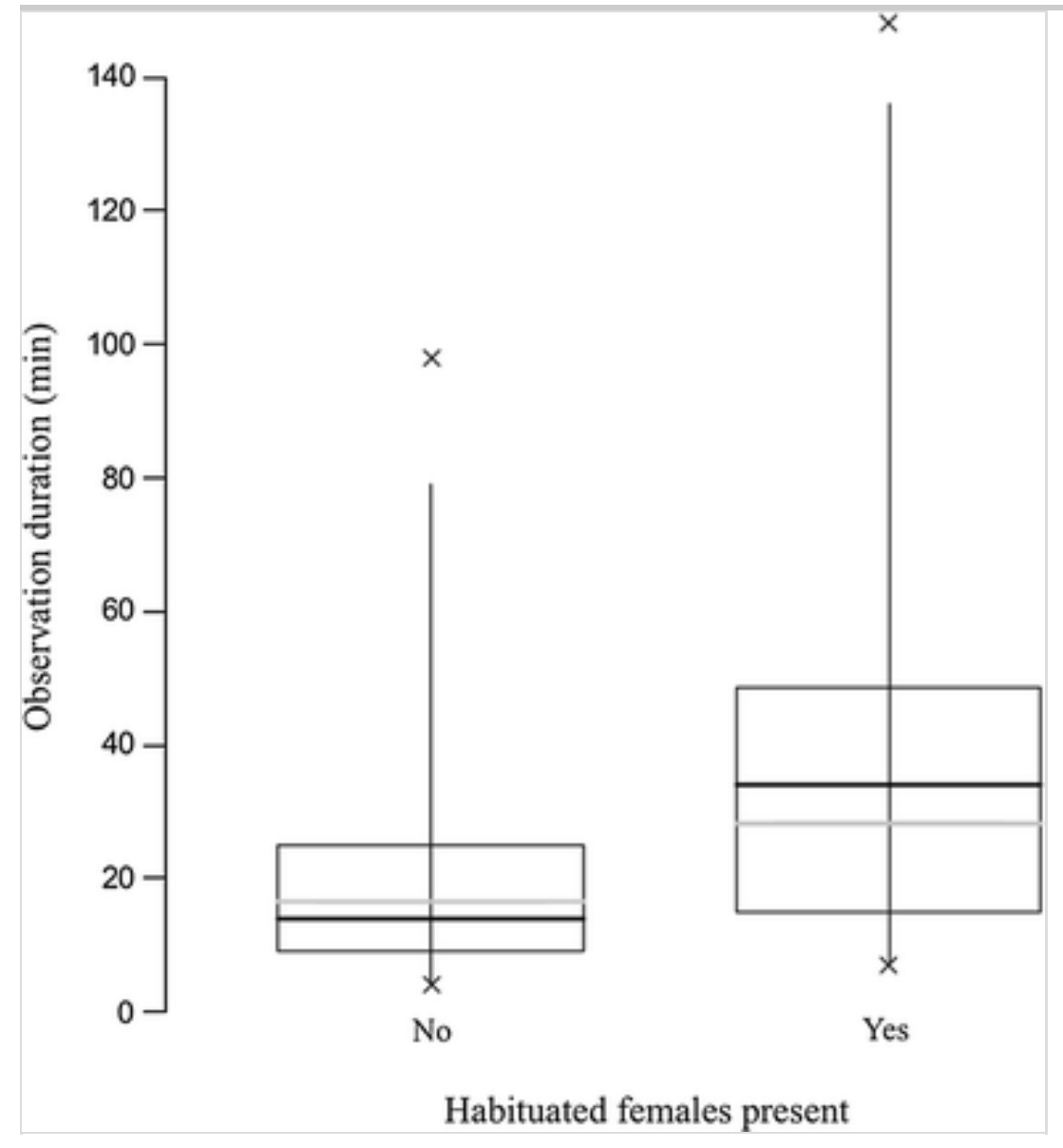

\section{Table 2}

Mean results (averaged across 1,000 random selections of individuals) of the GLMM with observation duration as the response and without the interaction between date and the presence of the habituated females

\begin{tabular}{|l|l|l|l|l|l|l|}
\hline Term & Estimate & SE & \multicolumn{1}{l|}{ t } & $\chi^{2}$ & df & P \\
\hline Intercept & 2.947 & 0.105 & 29.273 & a & a & a \\
\hline Presence $^{b}$ & 0.506 & 0.215 & 2.465 & 6.079 & 1.000 & 0.021 \\
\hline Date $^{c}$ & 0.060 & 0.103 & 0.583 & 0.459 & 1.000 & 0.526 \\
\hline Party size & 0.301 & 0.084 & 3.579 & 12.161 & 1.000 & 0.001 \\
\hline
\end{tabular}

${ }^{a}$ Not indicated because of having no meaningful interpretation

${ }^{b}$ Dummy coded $(0=$ habituated females absent; $1=$ habituated females present) and then centred to a mean of zero 
Excluding individuals one by one revealed the model results to be associated with considerable uncertainty. In fact, the $P$ values derived for the interaction between presence of the habituated females and date range from $<0.001$ to 1 , but also the results for the other terms in the model showed considerable variation (ESM Table S2).

\section{Discussion}

In this study, we present evidence that both the presence of the habituated individuals and the number of individuals in the group (party size) have an effect on the behaviour of non-habituated wild chimpanzees towards human observers. The presence of the habituated individuals was reflected in longer observation times and shorter observation distances between nonhabituated individuals and human observers compared to other periods. The effect of party size was more complex, with a positive relation between the number of individuals and observation time, although this also led to an increase in observation distance.

Both habituated females had been born into the Sonso community to habituated mothers, and therefore, when they transferred to Waibira at adolescence, they exhibited very high levels of tolerance to human observers, including resting on the ground within a few metres. In their presence, we were able to regularly observe non-habituated individuals on the ground at close proximity (Fig. 1) and for long-time periods (Fig. 2), irrespective of their age or sex. There appeared to be an interaction between the presence of these females and time lag from the onset of habituation, with an apparently stronger effect in the earlier stages of the habituation process.

When comparing the speed of habituation in the Waibira community with other habituation attempts, we observed a remarkable difference (first 2 years of regular observations: Waibira $=33 \%$; Tai North and Gashaka $<2 \%$; Boesch and Boesch 1990; Sommer et al. 2004). As at the two other sites, we never provisioned the chimpanzees with food and followed a habituation protocol very similar to Gashaka and near identical to that employed in Tai (Bertolani and Boesch 2008). It is possible that the relatively slow habituation rates at Gashaka can be partially explained by difficult terrain, but this is unlikely a sufficient explanation. Habituation of 
the Sonso community in Budongo was also much slower (Newton-Fisher 1999 ) than what we have-observed. As neighbours to the habituated Sonso community, Waibira chimpanzees would have had been occasionally exposed to the presence of human observers during intercommunity encounters. However, our data suggests that the presence of the two habituated females at Waibira was at least partly responsible for the dramatic difference in habituation speed. Their presence may have increased the likelihood that non-habituated individuals would remain in contact with the observers, thus acting as 'catalysts' for future encounters and increasing contact frequency, a necessary component in achieving habituation (Bertolani and Boesch 2008).

Party size also had a positive effect on observation time, but, counterintuitively, it was also positively related to observation distance between non-habituated individuals on the ground and human observers. We had initially expected that larger parties might reduce observer distance, due to the increased perceived security mediated by the number of individuals available to monitor the human observers. Although why observation distance should increase with group size remains unclear, it may be that the increase in observation duration (which occurred in large parties irrespective of the presence of the habituated females) is the result of a trade-off with increased distance. In other words, the group may stay longer because they remain further away. Another possibility is that the effect of the presence of the habituated females may become diluted with increasing numbers of unhabituated individuals and is thus diminished in the case of larger parties, leading to an increase in observation distance.

The ability to focal follow individuals at a close distance and for prolonged periods, without causing any interference in their natural behaviour, is a key element in the study of wild chimpanzees. We suggest therefore that when considering groups for habituation, the potential presence of the habituated individuals should be considered a key criterion. In addition, the tendency for larger groups to remain for longer periods in the presence of observers potentially facilitates both increasing the rate of habituation (through contact time) and more accurate data collection (through longer and more continuous periods of observation).

\section{Habituation as social learning}

Our results suggest that habituation to an initially aversive stimulus, the 
presence of human observers, can be mediated by a social learning process. As mentioned earlier, much of the current social learning literature is methodologically derived from Thorndike's puzzle box paradigm. From an evolutionary perspective, this is suboptimal, since wild animals, including non-human primates, spend very little time attempting to solve mechanical problems of this kind. Rather, the default case is that of younger, inexperienced individuals trying to learn something about the various aspects of their social and ecological environment through observing others.

For this reason, and although it may be a main learning mechanism in the wild, it is not surprising that one of the least studied areas of social learning is observational conditioning (encompassing effects also described as social referencing; Whiten et al. 2004). Observational conditioning or social referencing may also be particularly relevant for understanding habituation in the sense that they involve the ability to attribute another's behavioural response to an external event (Feinman et al. 1992; Whiten et al. 2004; Cheney and Seyfarth 2010). Most social animals appear to have specific communication signals, such as facial expressions, gestures, and vocalizations, that function as cues to reveal something about the attitude or inner state of the 'model' individual in relation to an external event. Thus, in the face of novel and initially aversive situations, such as the presence of human observers, the social environment may be crucial in facilitating learning.

Although examples of observational conditioning or social referencing in the wild are scarce, captive studies have revealed the relevance and importance of these mechanisms during the developmental stages of nonhuman primates. For example, in a classic study, Mineka et al. (1984) demonstrated that naïve captive rhesus monkeys showed no initial fear of snakes, but quickly learned to fear them by observing the responses of their (wild-born) parents. In a follow-up study, it was demonstrated that these learned responses were acquired more easily for biologically relevant than irrelevant stimuli. In particular, it was easier for a monkey to learn a fear response to a snake than to a flower (Cook and Mineka 1989). Infant chimpanzees were able to socially learn either a negative or a positive attitude towards a novel object, following a caregiver communicating 'fear' or 'happiness' towards the object by producing specific vocalizations and facial expressions (Russell et al. 1997). These studies indicate the capacity and significance of socially learned behaviour in non-human primates. 
We suggest that the change in behaviour of the non-habituated Waibira community members during habituation can be categorized as a two-step learning mechanism. Initially, the presence and neutral attitude of the two well-habituated females towards observers served as a 'model' from which the non-habituated community members learned socially to respond with less fear, facilitating longer and closer observations. The resulting increase in tolerance to and contact with human observers may then support a second step in the habitation process. This second step involves more direct acquisition of information about the human observers by non-habituated community members, in a non-associative learning process-habituation (i.e. a process that does not involve reward or punishment associated with stimulus). Opportunities for such information acquisition, such as particularly close-range and/or prolonged encounters, represent a fundamental element in promoting habituation. In the case of the Waibira community chimpanzees, the presence of the habituated females served as a catalyst for increased opportunities for information acquisition through social learning, promoting rapid and successful habituation.

Although it is very clear from the existing literature that social learning serves as a critical mechanism, through which both human infants (Barsalou et al. 2007) and wild animals (Galef and Laland 2004) learn about their environment, further empirical study is required. Given our findings and as trust and knowledge attribution to the 'model' are thought to facilitate social learning (Frith and Frith 2007), an interesting issue for future research would involve studying the behaviour of non-habituated females upon immigration into a research group where they are exposed to the behaviour of habituated individuals in the presence of humans on a daily basis.

\section{$\mathrm{AQ} 3$}

\section{Implications for conservation biology}

The habituation of free-living wild animals has become somewhat controversial due to evidence that it affects the ecology, health, and behaviour of the studied population; potentially involving serious risks of to both animals and humans (e.g. transmission of diseases, vulnerability to poachers; Woodford et al. 2002; Kasereka et al. 2006; Köndgen et al. 2008; Williamson and Feistner 2011), and must consequently be planned, monitored, and conducted with care. A particular issue is the possibility 
that habituated individuals will 'spread' their reduced fear of human observers to non-habituated populations (Goldsmith 2005). The presence of the habituated individuals within the Waibira community did affect the behaviour of non-habituated individuals, potentially providing them with a source of social reference while learning about the novel feature in their environment. However, the lengthy period of time needed to habituate individuals, even with the beneficial presence of the habituated females, suggests that the latter's presence facilitated rather than replaced the subsequent, necessary non-associative learning process of habituation. So while habituated individuals may act as a catalyst, speeding up habituation of a community, it is unlikely that, in chimpanzees, the simple transfer of habituated individuals to a non-habituated group would alone suffice to 'spread' habituation.

\section{Acknowledgments}

We would like to thank the Waibira chimpanzee field assistants, Simon Lokuyu, Gerald Mayanga, Gideon Atayo and Robert Eguma, and the group of international habituation volunteers; as well as to all the staff of the Budongo Conservation Field Station, the Uganda National Council for Science and Technology, the President's Office, the Uganda Wildlife Authority, and the National Forestry Authority. We thank Naomi Paz for her assistance in proofreading the manuscript. We also thank two anonymous reviewers for their thoughtful and helpful comments and one in particular for the discussion point that larger groups may dilute the effect of the habituated females. Fieldwork of $\mathrm{CH}$ and LS was funded by grants from the British Academy and a Leverhulme Trust's Research Leadership Award.

\section{Electronic supplementary material}

Below is the link to the electronic supplementary material.

Supplementary material 1 (DOCX $104 \mathrm{~kb})$

\section{References}

Akins CK, Zentall TR (1996) Imitative learning in male Japanese quail (Coturnix japonica) using the two-action method. J Comp Psychol 
Altmann J (1974) Observational study of behavior: sampling methods. Behaviour 49:227-267

Baar DJ, Levy R, Scheepers C, Tily HJ (2013) Random effects structure for confirmatory hypothesis testing: keep it maximal. J Mem Lang $68: 255-278$

Baayen RH (2008) Analyzing linguistic data. A practical introduction to statistics using R. Cambridge University Press, Cambridge

Barsalou LW, Breazeal C, Smith LB (2007) Cognition as coordinated non-cognition. Cogn Process 8:79-91

Bates D, Maechler M, Bolker B (2013) lme4: linear mixed-effects models using S4 classes. R package version 0.999999-2

Bertolani P, Boesch C (2008) Habituation of wild chimpanzees (Pan troglodytes) of the South Group at Tai Forest, Côte d'Ivoire: empirical measure of progress. Folia Primatol 79:162-171

Boesch C, Boesch H (1990) Tool use and tool making in wild chimpanzees. Folia Primatol 54:86-99

Burrell BD, Sahley CL (2001) Learning in simple systems. Curr Opin Neurobiol 11:757-764

Byrne RW, Byrne JME (1993) The complex lead-gathering skills of mountain gorillas (Gorilla g. beringei): variability and standardization. Am J Primatol 31:241-261

Caldwell CA, Whiten A (2002) Evolutionary perspectives on imitation: is a comparative psychology of social learning possible? Anim Cogn 5:193-2002

Cheney DL, Seyfarth RM (2010) Primate communication and human language: continuities and discontinuities. In: Kappeler P, Silk J (eds) Mind the gap: tracing the origins of human universals. Springer, Berlin, 
pp 283-298

Cook M, Mineka S (1989) Observational conditioning of fear to fearrelevant versus fear-irrelevant stimuli in rhesus monkeys. J Abnorm Psychol 98:448

Custance D, Whiten A, Fredman T (1999) Social learning of an artificial fruit task in capuchin monkeys (Cebus paella). J Comp Psychol 113:13

Dobson AJ (2002) An Introduction to generalized linear models. Chapman \& Hall/CRC, New York

Eggling WJ (1969) Observations on the ecology of the Budongo rain forest, Uganda. J Ecol 34:20-87

Feinman S, Roberts D, Hsieh KF, Sawyer D, Swanson D (1992) A critical review of social referencing in infancy. In: Feinman $S$ (ed) Social referencing and the social construction of reality in infancy. Plenum Press, New York, pp 15-54

Field A (2005) Discovering statistics using SPSS. Sage Publications, London

Forstmeier W, Schielzeth H (2011) Cryptic multiple hypotheses testing in linear models: overestimated effect sizes and the winner's curse. Behav Ecol Sociobiol 65:47-55

Fox B, Weisberg S (2011) An R companion to applied regression, 2nd edn. Sage, Thousand Oaks

Frith CD, Frith U (2007) Social cognition in humans. Curr Biol 17(16):R724-R732

Galef BG, Laland KN (2004) Social learning in animals: empirical studies and theoretical models. Bioscience 55:489-499

Ghiglieri MP (1984) The Chimpanzees of Kibale forest. Columbia University Press, New York 
Goldsmith ML (2005) Habituating primates for field study: ethical considerations for African great apes. In: Turner TR (ed) Biological anthropology and ethics. State University of New York Press, Albany, pp 49-64

Hamilton WD (1971) Geometry of the selfish herd. J Theor Biol 31(2):295-311

Heyes CM, Dawson GR (1990) A demonstration of observational learning in rats using a bidirectional control. Q J Exp Psychol 42:59-71

Hobaiter C, Byrne RW (2010) Able-bodied chimpanzees imitate a motor procedure used by a disabled individual to overcome handicap. PLoS One 5(8):e11959

Hurlbert SH (1984) Pseudoreplication and the design of ecological field experiments. Ecol Monogr 54:187-211

Jack KM, Lenz BB, Healan E, Rudman S, Schoof VAM, Fedigan L (2008) The effects of observer presence on the behavior of Cebus capucinus in Costa Rica. Am J Primatol 70:490-494

Johns B (1996) Responses of chimpanzees to habituation and tourism in the Kibale forest, Uganda. Biol Conserv 78:257-262

Kasereka B, Muhigwa JBB, Shalukoma C, Kahekwa JM (2006) Vulnerability of habituated Grauer's gorilla to paoching in the KahuziBiega National Park, DRC. Afr Stud Monogr 27:5-26

Kawai M (1965) Newly-acquired pre-cultural behaviour of the natural troop of Japanese monkeys on Koshima Islet. Primates 6:1-30

Köndgen S, Kühl H, N'Goran PK, Walsh PD, Schenk S, Ernst N et al (2008) Pandemic human viruses cause decline of endangered great apes. Curr Biol 18:260-264

Kosheleff VP, Anderson CNK (2008) Temperature's influence on the activity budget, terrestriality, and sun exposure of chimpanzees in the Budongo Forest, Uganda. Am J Phys Anthropol 139:172-181 
Mackintosh NJ (1987) Neurobiology, psychology and habituation. Behav Res Ther 25:81-97

Magurran AE, Girling S (1986) Predator recognition and response habituation in shoaling minnows. Anim Behav 34:510-518

McGrew WC, Tutin CEG (1978) Evidence for a social custom in wild chimpanzees. Man 13:234-251

Mineka S, Davidson M, Cook M, Keir R (1984) Fear of snakes in wild and lab-reared rhesus monkeys. J Abnorm Psychol 93:355-372

Morgan D, Sanz C (2003) Naive encounters with chimpanzees in the Goualougo triangle, Republic of Congo. Int J Primatol 24:369-381

Newton-Fisher NE (1999) The diet of chimpanzees in the Budongo Forest Reserve, Uganda. Afr J Ecol 37:344-354

Nishida T (1987) Local traditions and cultural transmission. In: Smuts BB, Cheney DL, Seyfarth RM, Wrangham RW, Struhsaker TT (eds) Primate societies. University of Chicago Press, Chicago, pp 462-474

Nishida T, Kawanaka K (1972) Inter-unit-group relationships among wild chimpanzees of the Mahale Mountains. Afr Stud 7:131-169

Petrinovich L, Patterson TL (1981) Field studies of habituation: IV. Sensitization as a function of the distribution and novelty of song playback to white-crowned sparrows. J Comp Physiol Psychol 95:805

Plumptre AJ, Behangana M, Davenport TRB, Kahindo C, Kityo R, Ndomba E et al. (2003) The biodiversity of the Albertine Rift, Albertine Rift technical reports No. 3, p 105

Pusey A (1979) Intercommunity transfer of chimpanzees in Gombe National Park. In: Hamburg D, McCown E (eds) The great apes. Benjamin/Cummings, Menlo Park, pp 405-427

Quinn GGP, Keough MJ (2002) Experimental design and data analysis for biologists. Cambridge University Press, Cambridge 
R Core Team (2013) R: a language and environment for statistical computing. R Foundation for Statistical Computing, Vienna

Raderschall CA, Magrath RD, Hemmi JM (2011) Habituation under natural conditions: model predators are distinguished by approach direction. J Exp Biol 214:4209-4216

Reynolds V (2005) The Chimpanzees of the Budongo Forest: ecology, behaviour, and conservation: ecology, behaviour, and conservation. Oxford University Press, Oxford

Romanes GJ (1882) Animal intelligence. Kegan Paul Trench \& Co., London

Russell CL, Bard KA, Adamson LB (1997) Social referencing by young chimpanzees (Pan troglodytes). J Comp Psychol 111:185

Schaller GB (1963) The mountain gorilla; ecology and behavior. University of Chicago Press. xvii, Chicago, p 431

Schielzeth H (2010) Simple means to improve the interpretability of regression coefficients. Method Ecol Evol 1:103-113

Sherry DF, Galef BG (1984) Cultural transmission without imitation: milk bottle opening by birds. Anim Behav 32:937-938

Sommer V, Adanu J, Faucher I, Fowler A (2004) Nigerian Chimpanzees (Pan troglodytes vellerosus) at Gashaka: 2 years of habituation efforts. Folia Primatol 75:295-316

Stein L (1966) Habituation and stimulus novelty: a model based on classical conditioning. Psychol Rev 73:352-356

Tennie C, Call J, Tomasello M (2006) Push or pull: imitation versus emulation in great apes and human children. Ethology 112:1159-1169

Thorndike EL (1898) Animal intelligence: an experimental study of the associative process in animals. Psychol Rev Monogr 2:551-553 
Thorpe W (1963) Learning and instinct in animals. Methuen, London

Tomasello M (1990) Cultural transmission in the tool use and communicatory signalling of chimpanzees? In: Parker S, Gibson K (eds) Language and Intelligence in monkeys and apes: comparative developmental perspectives. Cambridge University Press, Cambridge, pp 274-311

Tomasello M, Davis-Dasilva M, Camak L, Bard K (1987) Observational learning of tool-use by young chimpanzees. Primates 30:35-50

Tutin CEG, Fernandez M (1991) Responses of wild chimpanzees and gorillas to the arrival of primatologists: behaviour observed during habituation. In: Box HO (ed) Primates responses to environmental change. Chapman \& Hall, London, pp 187-197

Whiten A, Ham R (1992) On the nature and evolution of imitation in the animal kingdom: reappraisal of a century of research. Adv Stud Behav 21:239-283

Whiten A, Horner V, Litchfield CA, Marshall-Pescini S (2004) How do apes ape? Learn Behav 32:36-52

Williams J (1999) Female strategies and the reasons for territoriality in chimpanzees: lessons from three decades of research at Gombe. Ph.D. thesis, University of Minnesota

Williamson EA, Feistner ATC (2011) Habituating primates: processes, techniques, variables and ethics. In: Setchell JM, Curtis DJ (eds) Field and laboratory methods in primatology: a practical guide, 2 nd edn. Cambridge University Press, Cambridge, pp 25-39

Woodford MH, Butynski TM, Karesh WB (2002) Habituating the great apes: the disease risks. Oryx 36(02):153-160 